\title{
The cylindrical drift chamber for the MEG II
}

\author{
G. Chiarello $* a, b, c$ on behalf of the MEG collaboration \\ Author affiliation \\ a Istituto Nazionale di Fisica Nucleare Sezione di Lecce, Via Arnesano, Lecce, Italy \\ b Istituto Nazionale di Fisica Nucleare Sezione di Roma, Piazzale A. Moro, Roma, Italy \\ c Dipartimento Matematica e Fisica Ennio De Giorgi, Universitá del Salento, Lecce, Italy \\ E-mail: gianluigi.chiarello@le.infn.it
}

The MEG experiment, at PSI in Switzerland, aims at searching the charged lepton flavor violating decay $\mu^{+} \rightarrow e^{+} \gamma$. MEG has already determined the world best upper limit on the branching

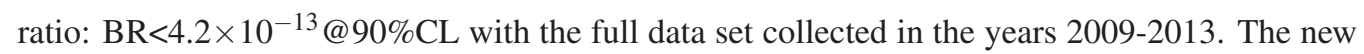
positron tracker is a high transparency single volume, full stereo cylindrical Drift Chamber (DC), immersed in a non uniform longitudinal B-field, co-axial to the muon beam line with length of $1.93 \mathrm{~m}$, internal radius of $17 \mathrm{~cm}$ and external radius of $30 \mathrm{~cm}$. It is composed of 10 concentric layers, divided in 12 identical sector of 16 drift cells each. The single drift cell is approximately square, with a $20 \mu \mathrm{m}$ gold plated W sense wire surrounded by $40 \mu \mathrm{m}$ silver plated Al field wires in a ratio of 5:1. For equalizing the gain of the innermost and outermost layers, two guard layers have been added at proper radii and at appropriate high voltages. The total number of wires amounts to 12288 for an equivalent radiation length per track turn of about $1.45 \times 10^{-3} \mathrm{X}_{0}$ when the chamber is filled with an ultra-low mass gas mixture of helium and iso-butane. Due to the high wire density $\left(12\right.$ wires $\left./ \mathrm{cm}^{2}\right)$, the use of the classical feed-through technique as wire anchoring system could hardly be implemented and therefore it was necessary to develop new wiring strategies. The number of wires and the stringent requirements on the precision of their position and on the uniformity of the wire mechanical tension impose the use of an automatic system (wiring robot) to operate the wiring procedures. Several tests have been performed in different prototypes of the drift chamber, exposed to cosmic rays, test beams and radioactive sources, to fulfill the requirement on the spatial resolution to be less than $110 \mu \mathrm{m}$. The drift chamber is currently under construction at INFN and should be completed by the end of 2017 to be then delivered to PSI.

EPS-HEP 2017, European Physical Society conference on High Energy Physics

5-12 July 2017

Venice, Italy

* Speaker. 


\section{The MEG Experiment}

The MEG experiment, at the Paul Scherrer Institute (PSI) near Zurich in Switzerland, aims at searching for the charged-lepton-flavor-violating decay $\mu^{+} \rightarrow e^{+} \gamma$, prohibited in the Model Standard but allowed, at a measurable level, in many of its extensions. With the analysis of the whole data collected in the years 2009-2013, which corresponds to $7.5 \times 10^{14} \mu^{+}$stopped on target, the upper limit on the branching ratio of $B R<4.2 \times 10^{-13}$ at $90 \%$ CL [1] was established. In order to improve the sensitivity by about one order of magnitude, MEG2, the upgrade of the MEG experiment was designed and approved [2] .

\section{The cylindrical drift chamber}

The positron tracker among other detectors, will have major improvements. It consists of a unique volume low-mass Cylindrical Drift CHamber (CDCH) [2] with high granularity and full stereo wires configuration. The CDCH radially extends from $R_{\text {inner }}=196 \mathrm{~mm}$ to $R_{\text {outer }}=284 \mathrm{~mm}$ and the active length are is $\sim 193 \mathrm{~cm}$ long. With the new tracking system layout one of the biggest advantage is that positrons with a momentum greater than $45 \mathrm{MeV} / \mathrm{c}$ will encounter a very small amount of material before reaching the timing counter (TC), $\lesssim 2.0 \times 10^{-3} X_{0}$, allowing to track the positrons trajectories up to as close as possible to the $\mathrm{TC}$, in order to minimize the contribution of the track length measurement to the positron timing resolution and to increase the positron reconstruction efficiency.

The CDCH is composed of 10 layers of drift cells at alternating stereo angles. Each layer has 192 square cells with ratio of field wires to sense wires equal to 5:1 to ensure the proper electrostatic configuration, each layers is composed by one anode sub-layers and 2 cathode sub-layers, see Fig. 1. The cell size varies linearly between $6.7 \mathrm{~mm}$, at the inner radius, and $8.7 \mathrm{~mm}$ at the outer radius, see Fig. 1. The alternating stereo angles vary from $5.8^{\circ}$ to $8.5^{\circ}$. The anodes are $20 \mu \mathrm{m}$ diameter gold plated tungsten wires, while the cathode are 40 and $50 \mu \mathrm{m}$ silver plated aluminum wires. To equalize the gain of the innermost and outermost layers, two layers of guard wires surround the active volume of the chamber. In total the $\mathrm{CDCH}$ is made with 1920 sense wires, 8448 field wires and 768 guard wires [3, 4].

The $\mathrm{CDCH}$ will be operated with a $85: 15 \mathrm{He} / \mathrm{i}-\mathrm{C}_{4} \mathrm{H}_{10}$ gas mixture, chosen for its low mass and its fast enough drift velocity.

\section{The construction of the drift chamber}

Due to the high wire density $\left(12\right.$ wires $\left./ \mathrm{cm}^{2}\right)$, the use of the traditional feed-through technique as wire anchoring system could hardly be implemented and therefore it was necessary to develop new wiring strategies. For the construction of the MEG-II CDCH, multi-wires frames (32 wires for the MEG-II case) were assembled by soldering the wires between two $400 \mu \mathrm{m}$ thick Printed Circuit Boards (called wire PCBs). The number of wires and the stringent requirements on the precision of their position and on the uniformity of the wire mechanical tension because of electrostatic wire stability impose the use of an automatic system (Wiring Robot), to operate the wiring procedures. 


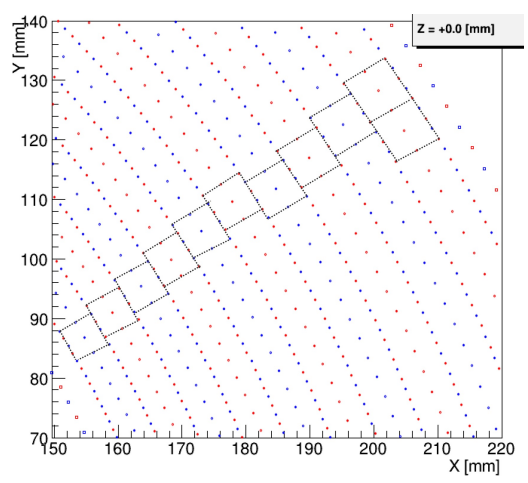

Figure 1: Drift cells configuration at the center of COBRA.

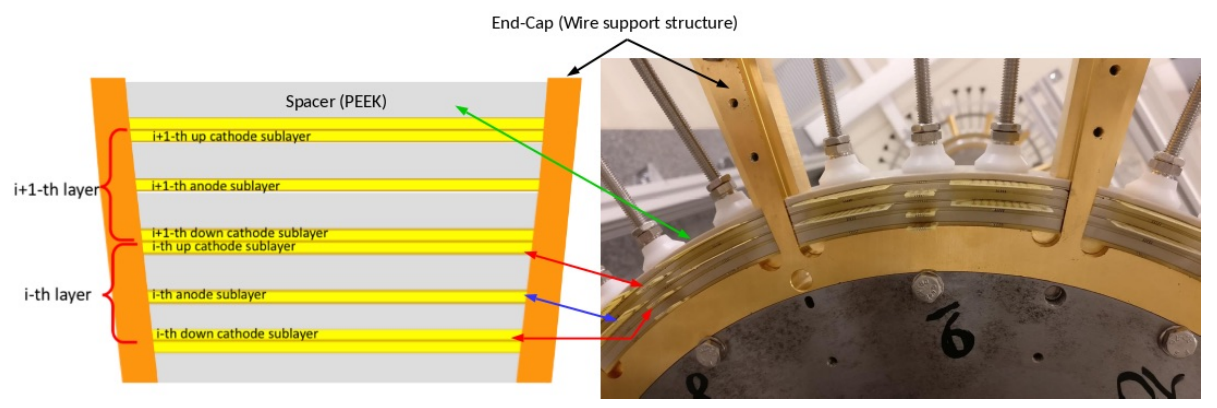

Figure 2: Schematic view (right) and real picture (left) of the stack of wire PCBs and spacers on the end-cap wiring support structure defining the drift chamber geometry.

\subsection{The wiring robot}

The wiring robot (Fig. 3) [5, 6] has been designed for:

- winding in a multi-wire frame a large number of densely spaced wires;

- applying to the wires the desired mechanical tension and maintaining it constant and uniform through the whole chamber;

- monitoring the wire positions and their alignments within a few tens $\mu \mathrm{m}$;

- fixing the wire on the PCB with a contact-less soldering system.

In order to fulfill all these constraints, the wiring robot, designed and built at the INFN Lecce and University of Salento laboratories, consists of:

- WIRING SYSTEM: the purpose of this system is the wiring of a multi-wire layer made up of 32 parallel wires at any stereo angle. In order to obtain a multi-wire layer, the wire PCB is positioned and aligned in the template on the winding cylinder and in a single operation by winding along a helical path the same wire multiple times around the cylinder with a pitch corresponding to the wire PCBs spacing. The mechanical tension to the wire is delivered by an electromagnetic brake, acting on the wire spool, and on-line monitored by a high precision dedicated system. The position of the wire is controlled, during the wiring phase, by a 


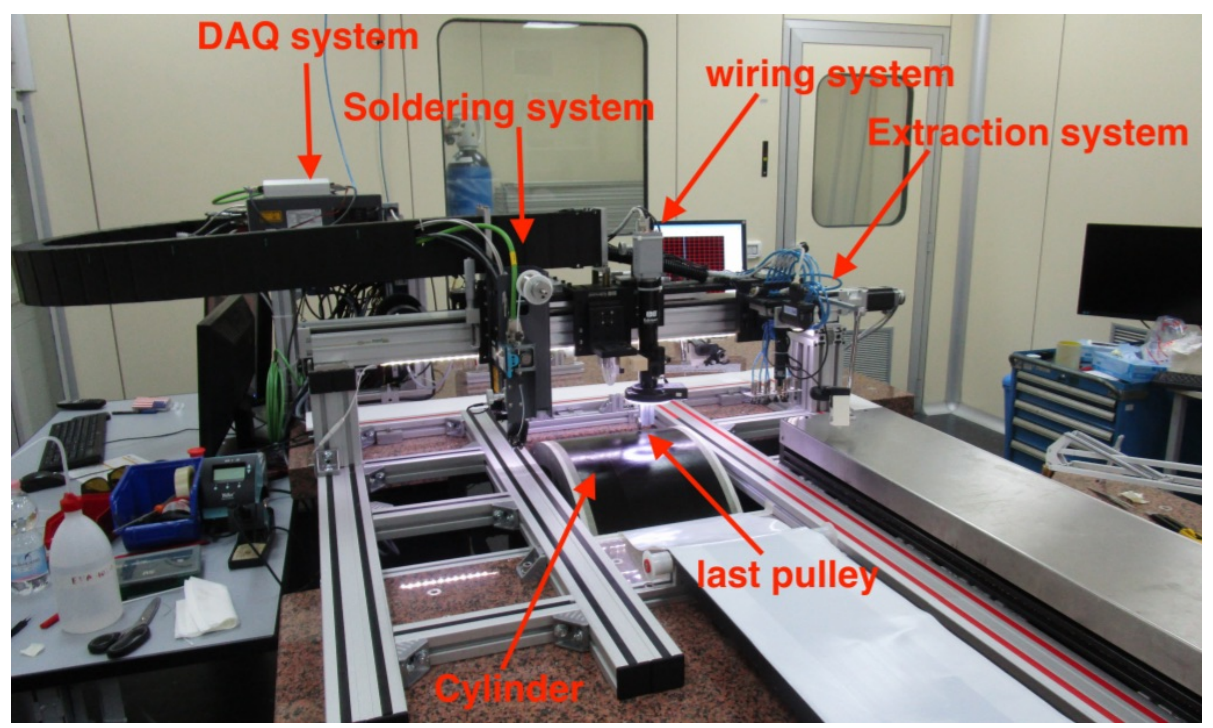

Figure 3: Wiring Robot.

digital camera and a linear slide, the accuracy on the position is $<20 \mu \mathrm{m}$ (Figure 4: left).The measured wire tension value is used in a real-time feedback system to correct the tension applied by the electromagnetic brake. Thanks to the feedback system the wire tension is delivered with a instantaneous variation of $0.15 \mathrm{~g}$ and a variations per single wire of less $\pm 0.05 \mathrm{~g}$ during a wiring sequence, see Fig. 4: right.

- SOLDERING SYSTEM: the soldering phase is implemented by an IR laser soldering system (LASCON Hybrid with a solder wire feeder [8]). Each wire is soldered at both ends while still constrained around the winding cylinder under its own tension. To avoid damages to the frail $\mathrm{Al}$ field wires, the soldering process is done at a temperature of $180^{\circ} \mathrm{C}$ by using a low melting point $\left(160^{\circ} \mathrm{C}\right)$ tin alloy. The laser system is controlled and synchronized with the positioning system that, by using a pattern matching software developed with LabVIEW, localize automatically the soldering pad.

- AUTOMATIC HANDLING: The wound layer of soldered wires around the cylinder must be unrolled and de-tensioned for storage and transport. This is accomplished with an automatic device. First, the wire is cut between the two wire PCB. Then, the first wire PCB is lifted off from the cylinder surface with a linear actuator connected to a set of vacuum operated suction cups and placed on the storage and transport frame. The unrolling is accomplished by synchronizing the cylinder rotation with the linear displacement of the frame. Once the layer of soldered wires is completely unrolled, the second wire PCB is lifted off from the cylinder, as the first one, and placed on the frame. After the extraction system, the multi-wire frames are examined and stored in sets of 13 (for a full layer of 12 sectors plus one spare). The 3 sets of 13 multi-wire frames, wrapped in a welded seal bag to avoid contamination from outside and equipped with valves in order to be flushed with dry gas, are then shipped to the assembly site in Pisa. 


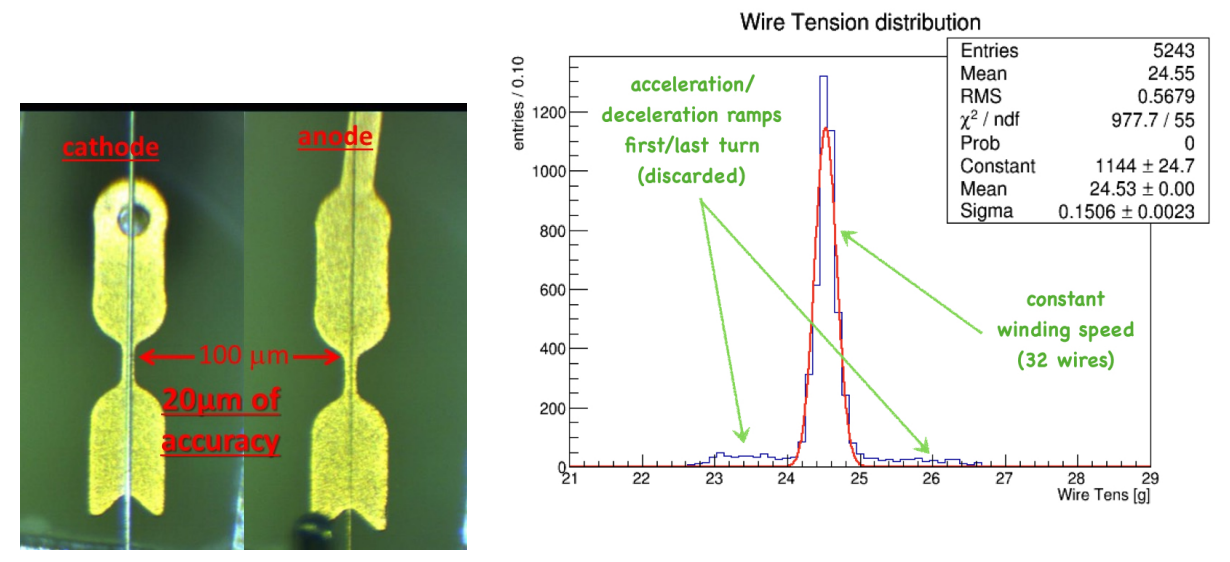

Figure 4: left: accuracy on the position of the wire. center: Tension measuring system. right: Strain gauge and anode wire tension variations

A real-time system, based on a National Instrument Compact RIO platform[7], controls the three systems simultaneously, sequencing and synchronizing all the different operations.

\subsubsection{Assembly procedure}

After passing the mechanical stretch test (a repeated cycle of elongation up to $25 \%$ over the nominal tension), the multi-wire frame can be mounted on the chamber end-plates. The wire of the frame must be kept parallel in order to avoid stresses to the wires at the soldering points. For this reason the assembly procedure is performed with a DEA Ghibli coordinate measuring machine. The machine allows a position accuracy of about $20 \mu \mathrm{m}$ in the horizontal plane and $40 \mu \mathrm{m}$ on the vertical axis of the fiducial markers on the wire PCBs. During the assembly phase, the end-plates (Figure 5: left above) are placed at a shorter than nominal distance to avoid stressing the wires.

The mounting procedure is performed by using an adjustable arm to release a multi-wire frame from the transport support and, by placing it next to the end-plates for the engagement procedure, to transfer the multi-wire frame on the end plates between two spokes. The final positioning is driven by hand through dedicated nippers. The wire PCBs are glued on the peek spacer with double-sided tape previously applied on the inner layer, see Fig. 2. This procedure is repeated for each of the 12 sectors and for the 10 layers.

After mounting the outermost layer, the end-plates will be moved to the nominal distance and the $\mathrm{CDCH}$ will be closed with the outer structural $2 \mathrm{~mm}$ thick carbon fiber cylinder. Then the endplates will be sealed to prevent gas leakage and the support structures (pcb holders, FE PCBs and extension) will be mounted prior to ship the CDCH to PSI.

\section{Conclusion}

The positron tracking system is one of the major improvements of the MEG upgrade, it will guarantee improvements of a factor: three on the positrons momentum, two on the angular resolution, two on acceptance and on tracking efficiency. These expected improvements due to a different layout are confirmed by the detailed simulation of the experiment. The performance of 


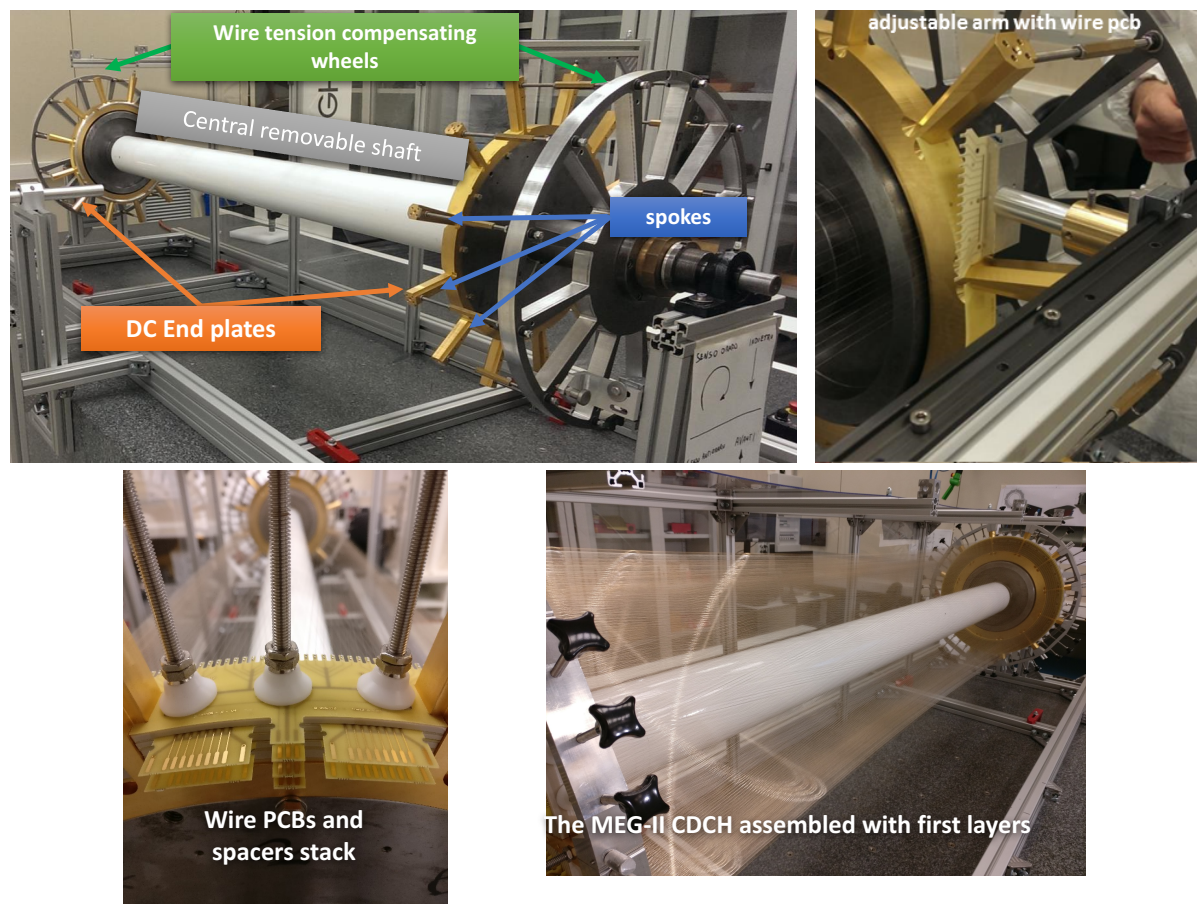

Figure 5: left above: DC assembly station. left below: PCB and spacer stack. right above: engagement procedure. right below: First layers mounted on the end plates.

the new positron spectrometer will contribute to improve MEG results by one order of magnitude.

\section{References}

[1] A.M. Baldini et al., Search for the Lepton Flavour Violating Decay $\mu^{+} \rightarrow e^{+} \gamma$ with the Full Dataset of the MEG Experiment ,Eur. Phys. J. C (2016) pg.76:434, arxiv:1605.05081[hep-phy].

[2] A. Baldini et al. (MEG Collaboration), MEG Upgrade Proposal, arxiv:1301.7225.

[3] A.M. Baldini et al., A new cylindrical drift chamber for the MEG II experiment, Nuclear Instruments and Methods in Physics Research Section A 824 (2016) pg.589-591,

DOI:10.1016/j.nima.2015.10.103.

[4] Chiarello G., The full stereo drift chamber for the MEG II experiment, JINST 12 (2017): C03062

[5] G. Chiarello et al., A new construction technique of high granularity and high transparency drift chambers for modern high energy physics experiments, Nuclear Instruments and Methods in Physics Research Section A 824 (2016) pg.512-514, DOI:10.1016/j.nima.2015.12.021

[6] G. Chiarello et al., A new assembly technique of full stereo Drift Chamber for high energy physics experiments, Advances in Sensors and Interfaces (IWASI) (2016), DOI:10.1109/IWASI.2015.7184938.

[7] National Instrument, The CompactRIO Platform - National Instruments, http://www.ni.com/compactrio/.

[8] Dr. Mergenthaler GmbH \& Co. KG, LASCON Hybrid System, http://www.ma-info.de/en/lascon-hybrid.html. 